\title{
Influence of longitudinal force and internal pressure on the frequency of free vibrations of an underground oil pipeline
}

\author{
Andrey Dmitriev ${ }^{1, *}$, Vladimir Sokolov ${ }^{1}$, and Aleksey Bereznyov ${ }^{1}$ \\ ${ }^{1}$ Industrial University of Tyumen, Volodarskogo str.38, Tyumen, 625001, Russian Federation
}

\begin{abstract}
This paper is based on the equation obtained earlier by V.G. Sokolov to find the frequencies of natural vibrations of straight sections of large-diameter pipelines. In this work, to take into account the effect of hydrostatic pressure on the pipeline wall from oil flowing at different speeds, the solution obtained by M.A. Ilgamov and A.S. Volmyr is used. At the same time, the effect of a stationary fluid flow on the pipeline wall is taken into account in the equation written in forces for the last term of the normal component of inertia forces. The resulting modified equation allows determining the frequency characteristics of the pipeline both according to the rod theory (without taking into account the deformation of the cross section) and according to the theory of shells (taking into account the deformation of the cross section).
\end{abstract}

\section{Introduction}

In the face of increased demand for products from oil refineries, the need for raw materials is increasing. In this regard, during the construction or reconstruction of a pipeline to ensure uninterrupted supply of the product, it becomes necessary to increase the pipe diameter over $1000 \mathrm{~mm}$ with a wall thinness parameter $(h / R=1 / 20-1 / 50)$. During the operation of these thin-walled oil and gas pipelines, the pipes are subjected to various kinds of dynamic impacts. Such an impact can be caused by vibrations from pumping equipment, seismic vibrations, as well as periodic vibrations caused, for example, by a train passing near the main oil and gas pipeline, as a result of wheel impacts on the rail joints.

The task of ensuring the reliability of the pipeline in conditions of dynamic impacts is to eliminate resonance phenomena, i.e. to determine the frequencies of natural and forced vibrations, depending on the physical, mechanical and geometric characteristics of the pipes. Thus, the natural frequency becomes one of the most important parameters when calculating the pipeline, along with the fluid flow rate, the geometric characteristics of the section, the internal working pressure, the rigidity of the subgrade, and the magnitude of the longitudinal compressive force. The analysis of pipelines for dynamic impact is currently an urgent problem. Studies to determine the frequency characteristics are reflected in the works [1 - 12], which consider straight pipe sections.

*Corresponding author: dmitrievav@tyuiu.ru 
During the construction of pipelines in the period from the $20 \mathrm{~s}$ to the $80 \mathrm{~s}$ of the last century, pipes with a diameter of less than $1000 \mathrm{~mm}$ with a wall thinness parameter $(\mathrm{h} / \mathrm{R}=$ $1 / 5-1 / 15)$ were used. These pipelines are calculated according to the methods based on the rod theory $[1-10]$, which does not take into account the deformation of the pipe crosssection. This approach is not applicable in the conditions of using pipes with a diameter of more than $1000 \mathrm{~mm}$, as, for example, during the construction of the Power of Siberia gas pipeline, in sections of which pipes with a cross section of $1720 \times 16 \mathrm{~mm}$ are used, which are difficult to characterize as a rod, therefore, it is not advisable to calculate these pipelines using methods based on the rod theory.

The fundamental concepts of the linear theory of small vibrations of thin-walled shells were laid down by A. Lyav. However, due to the cumbersomeness of the obtained equations, this solution did not find practical application. Therefore, to simplify the solution, a number of assumptions were subsequently used to obtain a practically applicable method for the dynamic calculation of cylindrical shells.

The most acceptable for practice is the theory of closed cylindrical shells. Among its variants, one can single out the most complete Flyuge theory, in which the forces of inertia of rotation are neglected for the equations proposed by Lyav. For a closed cylindrical shell with hinged ends, a solution is proposed in the form of a system consisting of three differential equations of motion in displacements. Solving these equations using Fourier series leads to a cubic equation for the square of the circular frequency of free flexural vibrations:

$$
a_{3} \omega^{6}+a_{2} \omega^{4}+a_{1} \omega^{2}+a_{0}=0
$$

This solution also turned out to be unsuitable for practical use due to its complexity. Therefore, the Flyuge equations of motion were subject to further simplification, in which the terms containing small factors with the squares of the ratio of the shell thickness $h$ to the radius of the median surface $\mathrm{R}$ were discarded. On the basis of such simplifications, a practically applicable and widely used Donnel-Mushtari-Vlasov equation was obtained, which is named by their authors (Donnel [13], Mushtari [14], Vlasov [15]). However, as was proved in the work of Ivanyuty and Finkil'shtejn [16], neglecting the tangential components of inertial forces, the values of the natural vibration frequencies of the shell are overestimated by $25 \%$.

For the first time, taking into account the influence of internal working pressure on the wall of a cylindrical shell when determining the frequencies of free vibrations can be traced in the works of Breslavski [17], Kukudzhanov [18, 19].

This work raises the question of a new approach to the dynamic calculation of thinwalled underground oil pipelines of large diameter, which is based on the application of the semi-momentless theory of shells of the average bend by Vlasov - Novozhilov [20, 21], in which the moments M1, which bend the cylindrical shell in the longitudinal direction, are neglected, since they are much less than the moments M2, which bend it in the transverse direction. The resolving equation of this approach is a homogeneous differential equation of the 4 th order, for the solution of which two boundary conditions are used at each end.

\section{Problem statement}

A cylindrical shell of finite length $L$, radius of the middle surface $R$ and wall thickness $h$, which is subject to the influence of constant internal working pressure p0, longitudinal compressive force $\mathrm{F}$, reaction of elastic soil resistance $\mathrm{q} 0$, soil pressure $\mathrm{qsl}=\mathrm{H} \gamma$, as well as the action of the velocity of the flowing liquid $\mathrm{V}$, was taken as a design scheme (figure 1). 


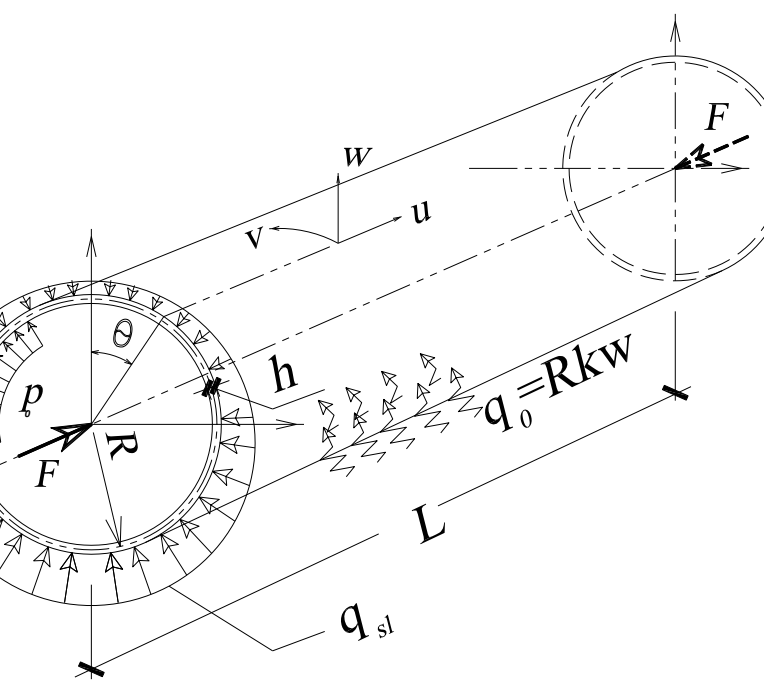

Fig.1. Design scheme of a pipeline.

To take into account the hydrostatic pressure qld created by the flow of oil flowing at a velocity V, the solution obtained by M.A. Il'gamov [23] and A.S. Vol'mir [24] is used:

$$
q_{l d}=\rho_{0} \Phi_{m n} \frac{R}{E h}\left(R^{2} \frac{\partial^{4} w}{\partial \theta^{2} \partial t^{2}}+V^{2} \frac{\partial^{4} w}{\partial \theta^{2} \partial \xi^{2}}\right)
$$

where $\rho 0$ - fluid density; $\Phi_{m n}=\frac{I_{m}\left(\lambda_{0}\right)}{\lambda_{0} I_{m}{ }^{\prime}\left(\lambda_{0}\right)}$ a parameter depending on the wave numbers in the circumferential and longitudinal directions $(m, n)$ and is determined by the ratio of the Bessel function to its derivative depending on $\lambda_{0}=\frac{n \pi R}{L}, \mathrm{~V}$ - fluid flow rate, product $\rho_{0} \cdot \Phi_{m n}$ - added fluid mass [23, 24].

\section{Building a solution}

To solve the problem on the frequency characteristics of an underground thin-walled oil pipeline of large diameter, the equation in forces obtained in [11] is used:

$$
\begin{gathered}
\frac{\partial^{2} T_{1}}{\partial \xi^{2}}+\frac{\partial}{\partial \xi}\left(\tau \frac{\partial M_{2}}{\partial \theta}\right)-\frac{\partial^{2}}{\partial \theta^{2}}\left(\frac{R_{2}^{*}}{R_{1}^{*}} T_{1}\right)-\frac{1}{R^{2}} \frac{\partial^{2}}{\partial \theta^{2}}\left(R_{2}^{*} \frac{\partial^{2} M_{2}}{\partial \theta}\right)-\frac{\partial}{\partial \theta}\left(\frac{1}{R_{2}^{*}} \frac{\partial M_{2}}{\partial \theta}\right)+ \\
+R \frac{\partial X_{1}}{\partial \xi}-R \frac{\partial X_{2}}{\partial \theta}--\frac{\partial^{2}}{\partial \theta^{2}}\left(R_{2}^{*} X_{3}\right)=0
\end{gathered}
$$

in this case, the effect of a stationary fluid flow on the pipeline wall is taken into account in the normal component of inertia forces X3:

$$
\begin{gathered}
X_{3}=-R h \rho \frac{\partial^{2} w}{\partial t^{2}}-\rho_{0} \Phi_{m n} \frac{R}{E h}\left(R^{2} \frac{\partial^{4} w}{\partial \theta^{2} \partial t^{2}}+V^{2} \frac{\partial^{4} w}{\partial \theta^{2} \partial \xi^{2}}\right)+p_{0}- \\
-\gamma H\left(2-\alpha_{1} \cos \theta-\alpha_{2} \cos 2 \theta\right)-\kappa R w
\end{gathered}
$$


Solving equation (3) using the assumptions of the semi-momentless theory of cylindrical shells $[11,20,21]$, after transformation, we obtain the differential equation of the pipeline motion in displacements:

$$
\begin{gathered}
\frac{\partial^{3} u}{\partial \xi^{3}}+\boldsymbol{h}_{v}^{2} \frac{\partial^{3}}{\partial \theta^{3}}\left(\frac{\partial^{2} \vartheta_{2}}{\theta}+\vartheta_{2}\right)+2 \frac{\partial^{2}}{\partial \theta^{2}}\left(\frac{\partial^{2} w}{\partial \xi^{2}} \varepsilon_{0}\right)-\frac{R}{E \boldsymbol{h}} p_{0} \frac{\partial^{3} \vartheta_{2}}{\partial \theta^{3}}+\frac{R^{2} w \kappa \cdot \partial^{2} w}{E \boldsymbol{h} \cdot \partial \theta^{2}}++R \gamma H \frac{\partial^{3} \vartheta_{2}}{\partial \theta^{3}}(2- \\
\left.\alpha_{1} \cos \theta-2 \cos 2 \theta\right)+R \gamma H\left(\frac{\partial^{2} \vartheta_{2}}{\partial \theta^{2}} \alpha_{1} \sin \theta+\frac{\partial^{2} \vartheta_{2}}{\partial \theta^{2}} 2 \alpha_{2} \sin 2 \theta\right)++R \gamma H\left(\frac{\partial \vartheta_{2}}{\partial \theta} \alpha_{1} \cos \theta-\right. \\
\left.\frac{\partial \vartheta_{2}}{\partial \theta} 4 \alpha_{2} \cos 2 \theta\right)-\frac{R^{2} \rho}{E}\left(\frac{\partial^{3} u}{\partial \xi \partial t^{2}}-\frac{\partial^{3} v}{\partial \xi \partial t}-\frac{\partial^{3} w}{\partial \theta^{2} \partial t^{2}}\right)+ \\
+\rho_{0} \Phi_{m n} \frac{R}{E \boldsymbol{h}}\left(R^{2} \frac{\partial^{4} w}{\partial \theta^{2} \partial t^{2}}+V^{2} \frac{\partial^{4} w}{\partial \theta^{2} \partial \xi^{2}}\right)=0
\end{gathered}
$$

where $\mathrm{u}, \mathrm{v}, \mathrm{w}$ - components of displacements of the middle surface of the shell, related to the radius $\mathrm{R}, 92$ - angle of rotation, p0 - internal pressure in the pipe, $\rho$ - soil lateral pressure coefficient, $\mathrm{H}$ - squeezed layer thickness, $\gamma$ - volumetric weight of soil, $\mathrm{E}-$ elastic modulus of pipe material, $\mathrm{R}-$ median surface radius, $h_{v}=\frac{h}{\left.R \sqrt{12\left(1-v^{2}\right.}\right)}-$ relative shell thickness parameter, $\mu \mathrm{bj}$ — added soil mass per unit of pipeline length, $\kappa$ coefficient of elastic soil resistance for a pipeline exposed to the action of internal working pressure [14], presented in the form:

$$
\kappa=\frac{E_{0}}{R\left(1+v_{0}\right)}
$$

The resulting system of equations (4) contains four unknown functions of coordinates and time t: $\mathrm{u}, \mathrm{v}, \mathrm{w}$, and 92 . Based on the Fourier method (method of separation of variables), we represent the function $\mathrm{w}(\xi, \theta, \mathrm{t})$, satisfying the condition of hinged support of the ends of the oil pipeline and periodicity along the circumferential coordinate $\theta$, in the form:

$$
w=\sum_{m} \cdot \sum_{n} b_{m n} \varphi(t) \sin \left(\overline{\lambda_{n}} \xi\right) \cos (m \theta)
$$

where $\overline{\lambda_{n}}=\frac{n \pi R}{L}, m, n=1,2 \ldots \ldots$ - wave numbers in the circumferential and longitudinal directions.

The rest of the displacement components and the angle of rotation $\$ 2$ are determined from the relations of the semi-momentless theory of shells given in [20, 21]:

$$
\begin{gathered}
u=-\sum_{m} \cdot \sum_{n} b_{m n} \frac{\overline{\lambda_{n}}}{m^{2}} \cos \left(\overline{\lambda_{n}} \xi\right) \cos (m \theta) ; \\
v=-\sum_{m} \cdot \sum_{n} b_{m n} \frac{1}{m} \sin \left(\overline{\lambda_{n}} \xi\right) \sin (m \theta) ; \\
\vartheta_{2}=-\sum_{m} \cdot \sum_{n} b_{m n} \frac{m^{2}-1}{m} \sin \left(\overline{\lambda_{n}} \xi\right) \sin (m \theta) .
\end{gathered}
$$

Taking into account that free vibrations of the shell move according to the harmonic law, we have:

$$
\varphi(t)=\sin \omega_{m n} t, \varphi^{\prime \prime}(t)=-\omega^{2} \sin \omega_{m n} t,
$$

where $\omega m n$ - first frequency of free bending vibrations in shape, $\mathrm{m}, \mathrm{n}=1,2,3 \ldots$ 
Substituting (7) - (9) into equation (5) and equating the coefficients at the same trigonometric functions $\cos (\mathrm{m} \theta)$ for $\mathrm{m}, \mathrm{n}=1,2,3 \ldots$, we obtain an infinite system of homogeneous linear algebraic equations with respect to the unknown amplitude values bmn of the radial component of the displacement $w$. The coefficients of the unknowns in these equations will be denoted by aij:

$$
\begin{aligned}
& \text { at } m=1 \quad a_{1,1} b_{1}+a_{1,2} b_{2}+a_{1,3} b_{3}=0 ; \\
& \text { at } m=2 \quad a_{2,1} b_{1}+a_{2,2} b_{2}+a_{2,3} b_{3}+a_{2,4} b_{4}=0 ; \\
& \text { at } m=3 \quad a_{3,1} b_{1}+a_{3,2} b_{2}+a_{3,3} b_{3}+a_{3,4} b_{4}+a_{5,5} b_{5}=0 .
\end{aligned}
$$

We write the resulting system of linear homogeneous algebraic equations in the form:

$$
\begin{gathered}
a_{m, m-2} b_{m-2, n}+a_{m, m-1} b_{m-1, n}+a_{m, m} b_{m, n}+ \\
+a_{m, m+1} b_{m+1, n}+a_{m, m+2} b_{m+2, n}=0
\end{gathered}
$$

where $\mathrm{m}=1,2,3 \ldots ; \mathrm{m} \pm 1>0 ; \mathrm{m} \pm 2>0$, and coefficients aij are defined by the expression:

$$
\begin{gathered}
a_{m, m}=A_{m, n}-B_{m, n} \omega_{m n}^{2} ; a_{m, m \pm 1}=-\frac{m^{5}(m \pm 2)}{2} q_{s l}^{*} \alpha_{1} ; a_{m, m \pm 2}=-\frac{m^{4}\left[(m \pm 2)^{2}-1\right]}{2} q_{s l}^{*} \alpha_{2} \\
A_{m, n}=\lambda_{n}^{4}+m^{4}\left(m^{2}-1\right)\left(m^{2}-1+p^{*}-2 q_{\mathrm{rp}}^{*}\right)+\kappa^{*} m^{4}-\lambda_{n}^{4} m^{4} P / n^{2} \\
B_{m, n}=\rho^{*} R h\left(\lambda^{2} h_{v}+m^{2}+m^{4}\right)+\mu_{b j}^{*} m^{4}+\rho_{0} \Phi_{m n} R^{2} m^{4}
\end{gathered}
$$

where $p^{*}=p_{0} \frac{R}{E h \cdot h_{v}^{2}} ; \rho^{*}=\rho_{0} \frac{R}{E h \cdot h_{v}^{2}} ; \kappa^{*}=\frac{R^{2} \kappa}{E h \cdot h_{v}^{2}} ; q_{\mathrm{rp}}^{*}=\frac{\gamma H R}{E h \cdot h_{v}^{2}} ; \lambda_{n}=\frac{n \pi R}{L \sqrt{h_{v}}} ;$

$P=\frac{F}{F_{\ni}}$; where $\mathrm{F}$ - longitudinal force; $F_{\ni}=\frac{\pi^{2} E I}{L^{2}}-$ Euler's force; $I=\pi R^{3} h-$ moment of inertia.

The coefficients of this system of equations (10) are dimensionless at the internal working pressure p0 in $\mathrm{mPa}$, the coefficient of elastic resistance $\kappa$ in $\mathrm{kN} / \mathrm{m} 3$, and the density of the shell material $\rho 0$ in $(\mathrm{kN} \cdot \mathrm{s} 2) / \mathrm{m} 4$.

The resulting system of homogeneous linear equations (11) is presented in matrix form:

$$
A \cdot B=\left[\begin{array}{cccccccc}
a_{11} & a_{12} & a_{13} & a_{14} & a_{15} & a_{16} & \ldots \ldots & a_{1 n} \\
a_{21} & a_{22} & a_{23} & a_{24} & a_{25} & a_{26} & \ldots \ldots & a_{2 p} \\
a_{31} & a_{32} & a_{33} & a_{44} & a_{45} & a_{46} & \ldots \ldots & a_{3 p} \\
a_{41} & a_{42} & a_{43} & a_{44} & a_{45} & a_{46} & \ldots \ldots & a_{4 p} \\
a_{51} & a_{52} & a_{53} & a_{54} & a_{55} & a_{56} & \ldots \ldots & a_{5 p} \\
a_{61} & a_{62} & a_{63} & a_{64} & a_{65} & a_{66} & \ldots \ldots & a_{6 p} \\
\ldots \ldots & \ldots \ldots & \ldots \ldots & \ldots \ldots & \ldots \ldots & \ldots \ldots & \ldots \ldots & \ldots \ldots \\
a_{p p-6} & a_{p p-5} & a_{p p-4} & a_{p p-3} & a_{p p-2} & a_{p p-1} & \ldots \ldots & a_{p p}
\end{array}\right] \cdot\left[\begin{array}{c}
b_{n 1} \\
b_{n 2} \\
b_{n 3} \\
b_{n 4} \\
b_{n 5} \\
b_{n 6} \\
\ldots \ldots \\
b_{n p}
\end{array}\right]=0 .
$$

Since the solution of this homogeneous system of linear algebraic equations is nonzero, because the magnitudes of the amplitude values of the radial displacement of the middle surface of the shell bmn $\neq 0$, the determinant of the coefficients of the homogeneous system (13) should be equal to zero: 


$$
|A|=\left|\begin{array}{cccccc}
A_{1 n}-B_{1 n} \omega^{2} & a_{12} & a_{13} & a_{14} & \ldots \ldots & a_{1 n} \\
a_{21} & A_{2 n}-B_{2 n} \omega^{2} & a_{23} & a_{24} & \ldots \ldots . & a_{2 n} \\
a_{31} & a_{32} & A_{3 n}-B_{3 n} \omega^{2} & a_{34} & \ldots \ldots & a_{3 n} \\
a_{41} & a_{42} & a_{43} & A_{4 n}-B_{4 n} \omega^{2} & \ldots \ldots & a_{4 n} \\
\ldots \ldots . & \ldots . . & \ldots \ldots . & \ldots \ldots & \ldots . . & \ldots \ldots . \\
a_{p-41} & a_{p-31} & a_{p-21} & a_{p-11} & \ldots . . & A_{p n}-B_{m n} \omega^{2}
\end{array}\right| \frac{1}{B_{1 n} B_{2 n} B_{3 n} B_{4 n} \ldots B_{m n}}=0
$$

After transformations, determinant (13) takes the form of the characteristic equation of matrix A:

$$
\left|\begin{array}{cccccc}
d_{11}-\lambda & d_{12} & d_{13} & d_{14} & \ldots \ldots & d_{1 n} \\
d_{21} & d_{22}-\lambda & d_{23} & d_{24} & \ldots \ldots & d_{2 n} \\
d_{31} & d_{32} & d_{33}-\lambda & d_{34} & \ldots \ldots & d_{3 n} \\
d_{41} & d_{42} & d_{43} & d_{44}-\lambda & \ldots \ldots & d_{4 n} \\
\ldots \ldots & \ldots \ldots & \ldots \ldots & \ldots \ldots & \ldots \ldots & \ldots \ldots \\
d_{p-41} & d_{p-31} & d_{p-21} & d_{p-11} & \ldots \ldots & d_{p n}-\lambda
\end{array}\right|=0
$$

where $d_{m, m}=\frac{a_{m, m}}{B_{m, n}} ; d_{m, m \pm 1} \frac{a_{m, m \pm 1}}{B_{m, n}} ; d_{m, m \pm 2} \frac{a_{m, m \pm 2}}{B_{m, n}} ; \lambda=\omega^{2}$ ( $\omega m n$ - frequency of free vibrations $(\mathrm{Hz}))$.

Coefficients Amn, Bmn, am, m+1, am, $\mathrm{m}+2$ are determined by formulas (12).

Solving determinant (15), we find the full spectrum of frequencies of free vibrations depending on the wave numbers $m$ and $n$, as well as the influence of the longitudinal force parameter, the value of the internal pressure, the coefficient of elastic resistance of the soil, the parameter of thinness, the added mass of the soil, the depth of the pipeline and the hydrostatic pressure oil.

\section{Results}

Let us analyze the influence of the internal working pressure, as well as the coefficient of elastic soil resistance $(\kappa)$ on the frequencies of free vibrations at fixed values of the parameter of the longitudinal force $\mathrm{P}$, the speed of movement of the oil product through the pipeline $\mathrm{V}$, the parameter of the length of the pipeline $\mathrm{L} / \mathrm{R}$ and the depth of the pipeline.

The obtained results shown in Table 1 and Fig. 2 allow drawing the following conclusions:

firstly, the minimum frequencies are realized for shell modes of vibration (taking into account the deformation of the cross section), i.e. for $m=2, n=1$;

secondly, with an increase in internal pressure, the frequency of free vibrations increases, which indicates an increase in the rigidity of the pipeline cross-section. Analysis of the results listed in Table 1 shows that the smaller the ratio $h / R$, the more sharply the frequency of free oscillations increases. For example, for the thinness parameter $h / R=1 / 30$ at an internal working pressure equal to zero and the coefficient of elastic soil resistance $\kappa=$ $0.04 \cdot 107 \mathrm{~N} / \mathrm{m} 3$, the frequency of free vibrations is $\omega 21=29.48 \mathrm{~Hz}$, and at $\mathrm{p} 0=5.0 \mathrm{mPa}$, frequency $\omega 21=45.93 \mathrm{~Hz}$, the growth rate was $36 \%$. For the thinness parameter $h / R=1 / 50$, other things being equal, the frequency $\omega 21$ increases from 14.66 to $31.94 \mathrm{~Hz}$, which corresponds to an increase of $45.9 \%$. The data on the increase in frequency characteristics are confirmed by the experiment described by the group of Brazilian scientists André Luiz Lupinacci Massa et al. [6], and the statement of Olav Fyrileiv [5] about the decrease in natural frequencies with increasing internal pressure is refuted.

thirdly, with an increase in the coefficient of elastic soil resistance, the frequencies of free vibrations increase, which also indicates an increase in the stiffness of the pipeline due 
to the fact that the soil prevents the movement of the walls of the thin-walled cross-section of the pipe. From the data given in Table 1 it can be seen that for a thin-walled pipe $\mathrm{h} / \mathrm{R}=1 / 50$ at zero internal working pressure and a longitudinal force parameter equal to zero, for $\kappa=0.04 \cdot 107 \mathrm{~N} / \mathrm{m} 3, \omega 21=14.66 \mathrm{~Hz}$, and at $\kappa=1.5 \cdot 107 \mathrm{~N} / \mathrm{m} 3, \omega 21=29.82 \mathrm{~Hz}$.

Table 1. Dependence of the frequency of free vibrations on the coefficient of elastic resistance of the soil and the internal working pressure.

\begin{tabular}{|c|c|c|c|c|c|c|c|c|c|}
\hline \multirow{4}{*}{$\begin{array}{c}\text { Frequency } \\
, \mathrm{Hz}\end{array}$} & \multicolumn{9}{|c|}{$p_{0}=0 \mathrm{mPa} ; P=0 ; V=3.0 \mathrm{~m} / \mathrm{s} ; L / R=10 ; H=2 \mathrm{~m}$} \\
\hline & \multicolumn{3}{|c|}{$h / R=1 / 30$} & \multicolumn{3}{|c|}{$h / R=1 / 40$} & \multicolumn{3}{|c|}{$h / R=1 / 50$} \\
\hline & \multicolumn{9}{|c|}{ Coefficient of elastic soil resistance $\mathrm{k} \cdot 10^{7} \mathrm{~N} / \mathrm{m}^{3}$} \\
\hline & 0.04 & 0.7 & 1.5 & 0.04 & 0.7 & 1.5 & 0.04 & 0.7 & 1.5 \\
\hline$\omega_{11}$ & 55.05 & 56.73 & 58.71 & 44.65 & 46.68 & 49.02 & 34.13 & 36.45 & 39.08 \\
\hline$\omega_{21}$ & 29.48 & 34.83 & 40.37 & 20.74 & 27.78 & 34.44 & 14.66 & 22.80 & 29.82 \\
\hline$\omega_{31}$ & 66.89 & 69.90 & 73.38 & 41.27 & 45.99 & 51.13 & 25.44 & 31.87 & 38.25 \\
\hline \multirow{4}{*}{$\begin{array}{c}\text { Frequency } \\
, \mathrm{Hz}\end{array}$} & \multicolumn{9}{|c|}{$p_{0}=3.0 \mathrm{mPa} ; P=0 ; V=3.0 \mathrm{~m} / \mathrm{s} ; L / R=10 ; H=2 \mathrm{~m}$} \\
\hline & \multicolumn{3}{|c|}{$h / R=1 / 30$} & \multicolumn{3}{|c|}{$h / R=1 / 40$} & \multicolumn{3}{|c|}{$h / R=1 / 50$} \\
\hline & \multicolumn{9}{|c|}{ Coefficient of elastic soil resistance $\mathrm{k} \cdot 10^{7} \mathrm{~N} / \mathrm{m}^{3}$} \\
\hline & 0.04 & 0.7 & 1.5 & 0.04 & 0.7 & 1.5 & 0.04 & 0.7 & 1.5 \\
\hline$\omega_{11}$ & 55.05 & 56.73 & 58.71 & 44.65 & 46.68 & 49.02 & 34.13 & 36.45 & 39.08 \\
\hline$\omega_{21}$ & 40.17 & 44.24 & 48.72 & 32.97 & 37.80 & 42.93 & 26.42 & 31.67 & 37.05 \\
\hline$\omega_{31}$ & 82.75 & 85.20 & 88.08 & 61.74 & 64.99 & 68.72 & 46.98 & 50.76 & 54.99 \\
\hline \multirow{4}{*}{$\begin{array}{c}\text { Frequency } \\
, \mathrm{Hz}\end{array}$} & \multicolumn{9}{|c|}{$p_{0}=5.0 \mathrm{mPa} ; P=0 ; V=3.0 \mathrm{~m} / \mathrm{s} ; L / R=10 ; H=2 \mathrm{~m}$} \\
\hline & \multirow{2}{*}{\multicolumn{3}{|c|}{$h / R=1 / 30$}} & \multicolumn{3}{|c|}{$h / R=1 / 40$} & \multicolumn{3}{|c|}{$h / R=1 / 50$} \\
\hline & & & & \multicolumn{6}{|c|}{ Coefficient of elastic soil resistance $\mathrm{k} \cdot 10^{7} \mathrm{~N} / \mathrm{m}^{3}$} \\
\hline & 0.04 & 0.7 & 1.5 & 0.04 & 0.7 & 1.5 & 0.04 & 0.7 & 1.5 \\
\hline$\omega_{11}$ & 55.05 & 56.73 & 58.71 & 44.65 & 46.68 & 49.02 & 34.13 & 36.45 & 39.08 \\
\hline$\omega_{21}$ & 45.93 & 49.54 & 53.58 & 39.05 & 43.20 & 47.76 & 31.94 & 36.40 & 41.17 \\
\hline$\omega_{31}$ & 91.82 & 94.03 & 96.64 & 72.23 & 75.03 & 78.28 & 56.99 & 60.14 & 63.75 \\
\hline
\end{tabular}
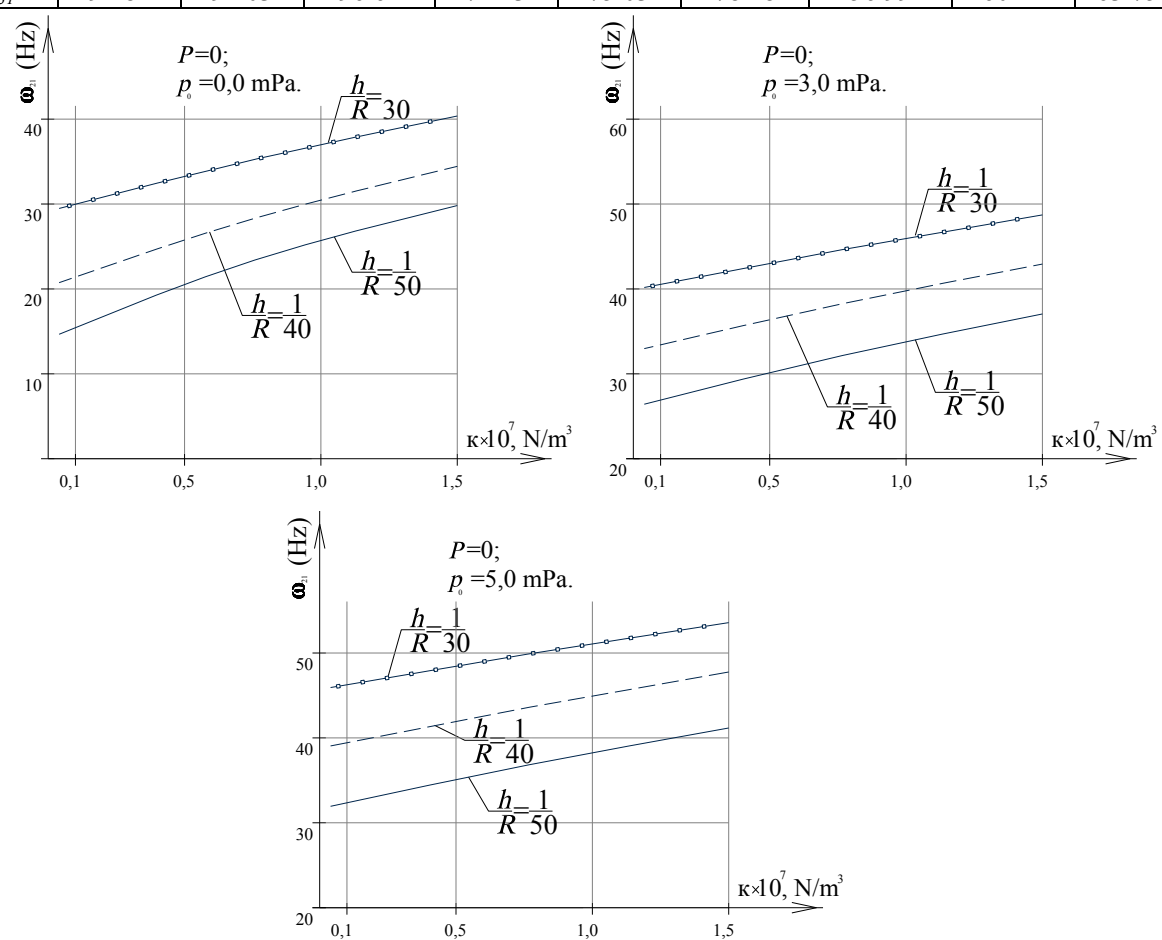

Fig. 2. Graphs of the dependence of the frequencies of free vibrations on the internal working pressure and the value of the coefficient of elastic soil resistance $(\kappa)$. 
Further, using (14), an analysis of the influence of the longitudinal force parameter on the frequencies of free vibrations of an underground oil pipeline at zero internal working pressure is carried out using different values of the coefficient of elastic soil resistance $(\kappa$ $\mathrm{N} / \mathrm{m} 3$ ) and different values of the thinness parameter (h/R). The results are shown in Table 2.

Table 2. Dependence of the frequency of free vibrations on the coefficient of elastic resistance of the soil, the internal working pressure and the parameter of the longitudinal force.

\begin{tabular}{|c|c|c|c|c|c|c|c|c|c|}
\hline \multirow{4}{*}{$\begin{array}{c}\text { Frequency } \\
, \mathrm{Hz}\end{array}$} & \multicolumn{9}{|c|}{$p_{0}=0 \mathrm{mPa} ; P=0.08 ; V=3.0 \mathrm{~m} / \mathrm{s}, L / R=10 ; H=2 \mathrm{~m}$} \\
\hline & \multicolumn{3}{|c|}{$h / R=1 / 30$} & \multicolumn{3}{|c|}{$h / R=1 / 40$} & \multicolumn{3}{|c|}{$h / R=1 / 50$} \\
\hline & \multicolumn{9}{|c|}{ Coefficient of elastic soil resistance $\mathrm{k} \cdot 10^{7} \mathrm{~N} / \mathrm{m}^{3}$} \\
\hline & 0.05 & 0.6 & 1.2 & 0.05 & 0.6 & 1.2 & 0.05 & 0.6 & 1.2 \\
\hline$\omega_{11}$ & 52.84 & 54.30 & 55.85 & 42.87 & 44.63 & 46.48 & 32.79 & 34.81 & 36.88 \\
\hline$\omega_{21}$ & 20.80 & 26.82 & 32.12 & 11.92 & 20.67 & 27.16 & 6.89 & 17.36 & 24.05 \\
\hline$\omega_{31}$ & 62.87 & 65.54 & 68.33 & 36.83 & 41.23 & 45.54 & 21.08 & 27.42 & 32.97 \\
\hline \multirow{4}{*}{$\begin{array}{c}\text { Frequency } \\
, \mathrm{Hz}\end{array}$} & \multicolumn{9}{|c|}{$p_{0}=0 \mathrm{mPa} ; P=0.13 ; V=3.0 \mathrm{~m} / \mathrm{s}, L / R=10 ; H=2 \mathrm{~m}$} \\
\hline & \multicolumn{3}{|c|}{$h / R=1 / 30$} & \multicolumn{3}{|c|}{$h / R=1 / 40$} & \multicolumn{3}{|c|}{$h / R=1 / 50$} \\
\hline & \multicolumn{9}{|c|}{ Coefficient of elastic soil resistance $\mathrm{k} \cdot 10^{7} \mathrm{~N} / \mathrm{m}^{3}$} \\
\hline & 0.05 & 0.6 & 1.2 & 0.05 & 0.6 & 1.2 & 0.05 & 0.6 & 1.2 \\
\hline$\omega_{11}$ & 51.39 & 52.89 & 54.49 & 41.70 & 43.51 & 45.40 & 31.89 & 33.97 & 36.09 \\
\hline$\omega_{21}$ & 12.51 & 21.05 & 27.49 & - & 15.62 & 23.55 & - & 13.92 & 21.70 \\
\hline$\omega_{31}$ & 60.19 & 62.97 & 65.87 & 33.70 & 38.46 & 43.05 & 17.72 & 24.93 & 30.94 \\
\hline \multirow{4}{*}{$\begin{array}{c}\text { Frequency } \\
, \mathrm{Hz}\end{array}$} & \multicolumn{9}{|c|}{$p_{0}=0 \mathrm{mPa} ; P=0.2 ; V=3.0 \mathrm{~m} / \mathrm{s}, L / R=10 ; H=2 \mathrm{~m}$} \\
\hline & \multicolumn{3}{|c|}{$h / R=1 / 30$} & \multicolumn{3}{|c|}{$h / R=1 / 40$} & \multicolumn{3}{|c|}{$h / R=1 / 50$} \\
\hline & \multicolumn{9}{|c|}{ Coefficient of elastic soil resistance $\mathrm{k} \cdot 10^{7} \mathrm{~N} / \mathrm{m}^{3}$} \\
\hline & 0.05 & 0.6 & 1.2 & 0.05 & 0.6 & 1.2 & 0.05 & 0.6 & 1.2 \\
\hline$\omega_{11}$ & 49.29 & 50.86 & 52.51 & 39.99 & 41.88 & 43.85 & 30.60 & 32.75 & 34.95 \\
\hline$\omega_{21}$ & - & 7.53 & 19.22 & - & 7.02 & 18.14 & - & 6.58 & 17.90 \\
\hline$\omega_{31}$ & 56.22 & 59.19 & 62.27 & 28.76 & 34.21 & 39.30 & 11.48 & 20.96 & 27.84 \\
\hline
\end{tabular}
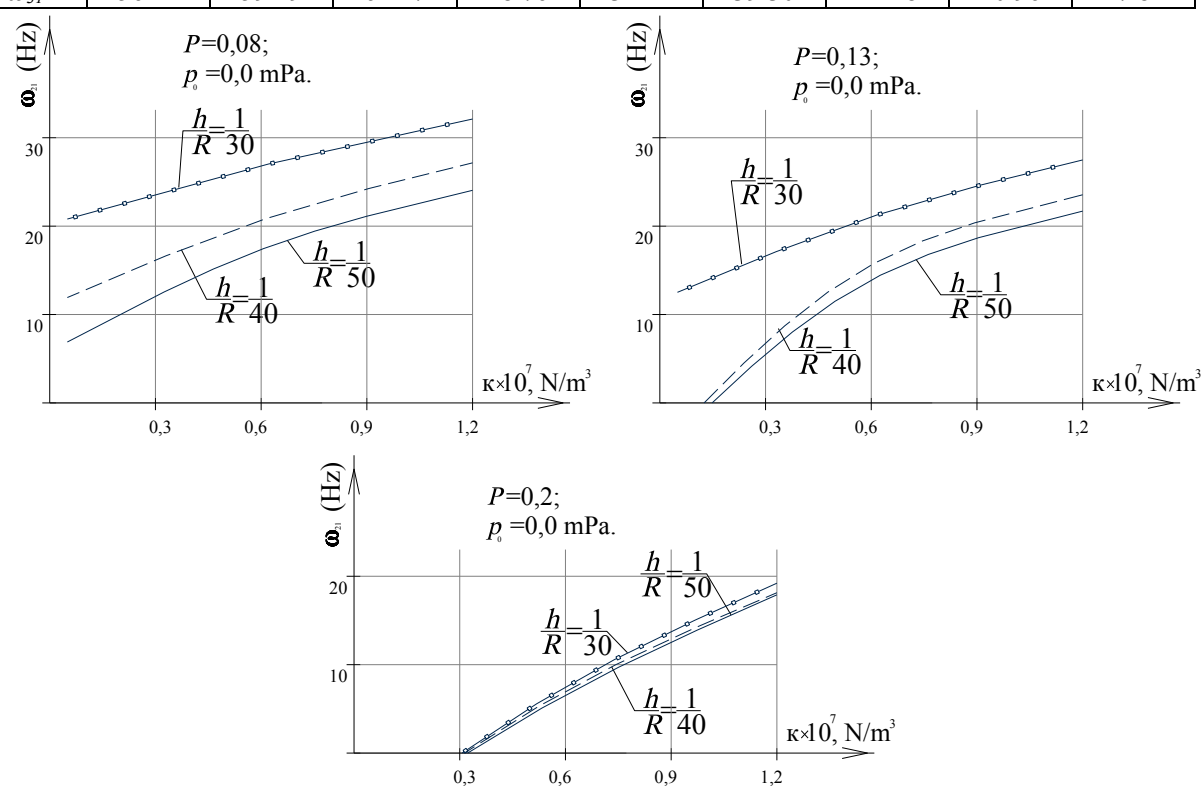

Fig. 3. Graphs of the dependence of the frequencies of free vibrations on the parameter of the longitudinal force and the value of the coefficient of elastic soil resistance $(\kappa)$.

The data given in Table 2 and Figure 3 show:

a) the minimum frequencies are realized for shell modes of vibration (taking into account the deformation of the cross section), i.e. for $\mathrm{m}=2, \mathrm{n}=1$; 
b) for oil pipelines with a thin-walled parameter $\mathrm{h} / \mathrm{R}=1 / 50, \kappa=0.6 \cdot 107 \mathrm{~N} / \mathrm{m} 3$, with zero internal pressure, with an increase in the longitudinal force parameter $\mathrm{P}$ from 0.08 to 0.2 , the frequency decreases by $37.9 \%$ from $17.36 \mathrm{~Hz}$ to $6.58 \mathrm{~Hz}$, and for the thinness parameter $\mathrm{h} / \mathrm{R}=1 / 30$ - by $28.1 \%$ from $26.82 \mathrm{~Hz}$ to $7.53 \mathrm{~Hz}$ (see Table 2, Figure 3).

c) for free-flow oil pipelines with the value of the thin-walled parameter $h / R=1 / 50$, laid in the ground with a small value of the coefficient of elastic soil resistance $\kappa=0.05 \cdot 107$ $\mathrm{N} / \mathrm{m} 3$, with an increase in the longitudinal force parameter $\mathrm{P}$ from 0.08 to 0.13 , the frequency (at $\mathrm{m}=2, \mathrm{n}=1$ ) sharply decreases from $6.89 \mathrm{~Hz}$ and can turn to 0 , i.e. a local loss of stability (flattening of the cross section) occurs.

\section{Conclusion}

The calculated data showed that the minimum frequency is realized for shell vibration modes $(m=2, n=1)$, taking into account the deformation of the cross section.

The internal working pressure significantly affects the natural frequency of a thinwalled pipeline, and the higher the pressure, the higher the frequency.

As the longitudinal compressive force increases, the frequency response decreases. At zero internal pressure for $\mathrm{P} \geq 0.15$ in soils with a coefficient of elastic resistance less than $0.05 \cdot 107 \mathrm{~N} / \mathrm{m} 3$, the frequency decreases and can turn to zero, which indicates the loss of stability of the cross section (flattening).

\section{References}

1. V.V. Bolotin, Vibrations and stability of an elastic cylindrical shell in the flow of a compressible liquid, Engineering collection 24, 3 - 16 (1956)

2. Ya.G. Panovko, Stability and vibrations of elastic systems: Modern concepts, paradoxes and errors (M.: KomKniga, 2007)

3. V.I. Feodos'ev, About fluctuations and stability of a pipe when a liquid flows through it, Eng. collector 10, 169 - 170 (1952)

4. G.V. Denisov, Proposals for the calculation of sections of buried main pipelines with structural inclusion for seismic impact, Transportation and storage of petroleum products and raw hydrocarbons 3, 20-23 (2013)

5. O. Fyrileiv, Effect of Internal Pressure on Free Spanning Pipelines (2010) 10.1115/IPC2010-31622

6. A. Massa, N. Galgoul, N. Junior, A. Fernandes, F. Coelho, S. Neto, The Influence of Internal Pressure on Pipeline Natural Frequency (2009) 10.1115/OMAE2009-79666

7. S. Georgiadou, E. Loukogeorgaki, D. Angelides, Dynamic analysis of a free span offshore pipeline, Proceedings of the International Offshore and Polar Engineering Conference 80-87 (2014)

8. E. Ruocco, R. Di Laora, V. Minutolo, An Exponential Matrix Method for the Buckling Analysis of Underground Pipelines Subjected to Landslide Loads, Procedia Earth and Planetary Science 16, 25-34 (2016) 10.1016/j.proeps.2016.10.004

9. W.-H. Xü, W.-D. Xie, X.-F. Gao, Y.-X. Ma, Study on vortex-induced vibrations (VIV) of free spanning pipeline considering pipe-soil interaction boundary conditions, Journal of Ship Mechanics 22, 446-453 (2018) 10.3969/j.issn.1007-7294.2018.04.007

10. G.P. Kourctzis, S.W. Sloan, Analysis of buried pipelines subjected to ground surface settlement and heave (2014) [Electronic resource]: vwvw.nrcrcsearchprcss.com/cgj 
11. V.G. Sokolov, Free vibrations of underground rectilinear thin-walled sections of gas pipelines, Bulletin of civil engineers 2 (73), 29-34 (2019)

12. I.O. Razov, Parametric fluctuations and dynamic stability of large- diameter oil trunk pipelines during ground laying, Bulletin of civil engineers 4 (45), 58-62 (2014)

13. L.H. Donnel, A new theory for the buckling of thin cylinders under axialcompression and bending, Trans. ASME. 56, 11, 86 - 94 (1934)

14. X.M. Mushtari, Nonlinear theory of elastic shells (Kazan': Tatknigoizdat, 1957)

15. V.Z. Vlasov, General theory of shells and its applications in engineering (M.: Gostekhizdat, 1949)

16. E.I. Ivanyuta, On the influence of tangential inertia forces on the frequency of free vibrations of a thin cylindrical shell, Research on elasticity and plasticity 2, $212-215$ (1963)

17. V.E. Breslavskij, Natural vibrations of a circular cylindrical shell under hydrostatic pressure, Izvestia of the USSR Academy of Sciences 12, 117 - 120 (1956)

18. S.N. Kukudzhanov, On the influence of normal pressure on the natural oscillation frequencies of cylindrical shells, Solid mechanics 3, 14-20 (1968)

19. S.N. Kukudzhanov, Vibrations and dynamic stability of rotation shells that are close to cylindrical, under the influence of normal pressure and meridional forces, WPI. Mechanics of Solids 2, 48-59 (2006)

20. V.Z. Vlasov, General theory of shells and its applications in engineering (M.: Gostekhizdat, 1949)

21. V.V. Novozhilov, The theory of thin shells (L.: Sudpromgiz, 1962)

22. V.P. Il'in, Application of semi-instantaneous theory to problems of calculation of thinwalled pipes, Problems of calculating spatial structures. Works of MISI 1, 45-55 (1980)

23. M.A. Il'gamov, Vibrations of elastic shells containing liquids and gases (M.: Nauka, 1969)

24. A.S. Vol'mir, Vibrations of the shell with a flowing liquid, Izv. ANSSSR MTT 6, 162 -166 (1973) 